REPRESENTATION THEORY

An Electronic Journal of the American Mathematical Society

Volume 15, Pages 574-583 (August 2, 2011)

S 1088-4165(2011)00404-0

\title{
SEMISTABLE LOCUS OF A GROUP COMPACTIFICATION
}

\author{
XUHUA HE AND JASON STARR
}

\begin{abstract}
In this paper, we consider the diagonal action of a connected semisimple group of adjoint type on its wonderful compactification. We show that the semistable locus is a union of the $G$-stable pieces and we calculate the geometric quotient.
\end{abstract}

0.1. Introduction. Let $G$ be a connected, semisimple algebraic group of adjoint type over an algebraically closed field and $X$ its wonderful compactification. We will give an explicit description of the semi-stable locus of $X$ (for the diagonal $G$-action) using Lusztig's $G$-stable pieces and calculate the geometric quotient $X / / G$. We also deal with the case where the $G$-action is twisted by a diagram automorphism.

0.2. In 3 De Concini, Kannan and Maffei described the semi-stable locus and geometric quotient for complete symmetric varieties (which includes as a special case the nontwisted conjugation action of $G$ on its wonderful compactification). Their approach is based on the study of the $G$-invariants of the Cox ring $R(X)$. Our approach here is totally different and is based on the geometry of the nilpotent cone of $X$. This allows us to give an explicit description of the semistable locus as a union of Lusztig's G-stable pieces. Such explicit description plays an important role in the study of character sheaves on the wonderful compactification [9]. Our approach also allows us to study the twisted action on the wonderful compactification.

0.3. Geometric invariant theory. The foundations of geometric invariant theory are developed in [13. We quickly review the part which we use. Let $k$ be a field. The setup for geometric invariant theory over $k$ consists of $(G, X, \tau, \mathcal{L}, \psi)$ where

(i) $G$ is a reductive algebraic group over $k$,

(ii) $X$ is a separated, finite type $k$-scheme,

(iii) $\tau: G \times X \rightarrow X$ is an algebraic action of $G$ on $X$,

(iv) $\mathcal{L}$ is an invertible sheaf on $X$, and

(v) $\psi: \tau^{*} \mathcal{L} \rightarrow \operatorname{pr}_{X}^{*} \mathcal{L}$ is a $G$-linearization of $\mathcal{L}$ (where $\operatorname{pr}_{X}: G \times X \rightarrow X$ is the projection), i.e., an isomorphism of invertible sheaves on $G \times X$ which defines a lifting of the action $\tau$ to an action of $G$ on $\operatorname{Spec}_{X} \operatorname{Sym}^{\bullet}(\mathcal{L})$.

The fundamental theorem of geometric invariant theory, 13 , Theorem 1.10, p. 38], associates to this datum a pair $\left(X^{\mathrm{ss}}(\mathcal{L}), \phi\right)$. Here $X^{\mathrm{ss}}(\mathcal{L})$ is the union $X_{s}$

Received by the editors January 28, 2009 and, in revised form, January 24, 2011.

2010 Mathematics Subject Classification. Primary 14L30, 14L24.

The first author was partially supported by (USA) NSF grant DMS 0700589 (HK) RGC grant DAG08/09.SC03 and RGC grant 601409.

The second author was partially supported by an Alfred P. Sloan fellowship, NSF grant DMS0553921 and NSF grant DMS-0758521.

(C)2011 American Mathematical Society Reverts to public domain 28 years from publication 
over all positive integers $n$ and all $G$-invariant sections $s$ of $\Gamma\left(X, \mathcal{L}^{\otimes n}\right)$, provided $X_{s}$ is affine (recall that $X_{s}$ is defined to be the maximal open subscheme of $X$ on which $s$ is a generator of $\left.\mathcal{L}^{\otimes n}\right)$. And $\phi$ is a $G$-invariant $k$-morphism

$$
\phi: X^{\mathrm{ss}}(\mathcal{L}) \rightarrow X / / \mathcal{L} G
$$

which is a uniform categorical quotient of the action of $G$ on $X^{\mathrm{ss}}(\mathcal{L})$. Moreover, the following hold.

(i) The morphism $\phi$ is affine and universally submersive.

(ii) For some integer $n>0$, there exists an ample invertible sheaf $\mathcal{M}$ on $X / /{ }_{\mathcal{L}} G$ such that $\phi^{*} \mathcal{N}$ is isomorphic to $\mathcal{L}^{\otimes n}$ as $G$-linearized invertible sheaves (in particular, $X / / \mathcal{L} G$ is quasi-projective).

(iii) There exists a unique open subscheme $U$ of $X / /_{\mathcal{L}} G$ such that $\phi^{-1}(U)$ is the stable locus. And the induced morphism $\phi: \phi^{-1}(U) \rightarrow U$ is a uniform geometric quotient of $\phi^{-1}(U)$.

Since we do not make use of them, we will not make precise the definitions of uniform categorical quotient, stable locus and uniform geometric quotient, but we will use a few other known facts about geometric invariant theory.

Fact 1 . When $X$ is projective and $\mathcal{L}$ is ample, every open $X_{s}$ is affine. Thus $X / /{ }_{\mathcal{L}} G$ is canonically isomorphic to

$$
X / /_{\mathcal{L}} G=\operatorname{Proj} \oplus_{n \geq 0} \Gamma\left(X, \mathcal{L}^{\otimes n}\right)^{G}
$$

and $X^{\mathrm{ss}}(\mathcal{L})$ is the maximal open subscheme of $X$ on which the natural rational map from $X$ to $X / / \mathcal{L} G$ is defined, [15].

Fact 2. Again, when $X$ is proper, every $G$-orbit $O$ in $X^{\text {ss }}(\mathcal{L})$ contains a unique closed $G$-orbit in its closure (in $X^{\mathrm{ss}}(\mathcal{L})$ ), and two $G$-orbits $O_{1}$ and $O_{2}$ in $X^{\mathrm{ss}}(\mathcal{L})$ are in the same fiber of $\phi$ if and only if the associated closed $G$-orbits are equals. In particular, $\phi$ establishes a natural bijection between the points of $X / /_{\mathcal{L}} G$ and the closed $G$-orbits in $X^{\text {ss }}(\mathcal{L})$, $[15$.

Fact 3 (Matsushima's criterion). A $G$-orbit $O$ is affine if and only if the stabilizer group of one (and hence every) closed point is itself reductive, 14. In particular, since the fibers of $\phi$ are affine, every closed $G$-orbit in $X^{\mathrm{ss}}(\mathcal{L})$ is affine, and hence has reductive stabilizer group.

Fact 4. If $X$ is normal (or if $X^{\mathrm{ss}}(\mathcal{L})$ is normal), then $\phi$ factors through the normalization of the target. Thus by the universal property, the target $X / /_{\mathcal{L}} G$ is normal.

0.4. Notations. Now we fix the notations used in the rest of this article. Let $G$ be a connected semisimple algebraic group of adjoint type over an algebraically closed field $k$. Let $B$ be a Borel subgroup of $G, B^{-}$an opposite Borel subgroup, and $T=B \cap B^{-}$. Let $\left(\alpha_{i}\right)_{i \in I}$ be the set of simple roots determined by $(B, T)$. We denote by $W$ the Weyl group $N(T) / T$. For $w \in W$, we choose a representative $\dot{w}$ in $N(T)$. For $i \in I$, we denote by $\omega_{i}, \omega_{i}^{\vee}$ and $s_{i}$ the fundamental weight, fundamental coweight and the simple reflection corresponding to $\alpha_{i}$.

For $J \subset I$, let $P_{J} \supset B$ be the standard parabolic subgroup defined by $J$ and let $P_{J}^{-} \supset B^{-}$be the parabolic subgroup opposite to $P_{J}$. Set $L_{J}=P_{J} \cap P_{J}^{-}$. Then $L_{J}$ is a Levi subgroup of $P_{J}$ and $P_{J}^{-}$. The semisimple quotient of $L_{J}$ of adjoint type will be denoted by $G_{J}$. We denote by $\pi_{P_{J}}\left(\right.$ resp. $\pi_{P_{J}^{-}}$) the projection of $P_{J}$ (resp. 
$\left.P_{J}^{-}\right)$onto $G_{J}$. Let $W_{J}$ be the subgroup of $W$ generated by $\left\{s_{j} \mid j \in J\right\}$ and $W^{J}$ the set of minimal length coset representatives of $W / W_{J}$.

0.5 . Wonderful compactification of $G$. We consider $G$ as a $G \times G$-variety by left and right translation. Then there exists a canonical $G \times G$-equivariant embedding $X$ of $G$ which is called the wonderful compactification (4, 21]). The variety $X$ is an irreducible, smooth projective $(G \times G)$-variety with finitely many $G \times G$ orbits $Z_{J}$ indexed by the subsets $J$ of $I$. The boundary $X-G$ is a union of smooth divisors $\overline{Z_{I-\{i\}}}$ (for $i \in I$ ), with normal crossing. The $G \times G$-variety $Z_{J}$ is isomorphic to the product $(G \times G) \times{ }_{P_{J}^{-} \times P_{J}} G_{J}$, where $P_{J}^{-} \times P_{J}$ acts on $G \times G$ by $(q, p) \cdot\left(g_{1}, g_{2}\right)=\left(g_{1} q^{-1}, g_{2} p^{-1}\right)$ and on $G_{J}$ by $(q, p) \cdot z=\pi_{P_{J}^{-}}(q) z \pi_{P_{J}}(p)^{-1}$. We denote by $h_{J}$ the image of $(1,1,1)$ in $Z_{J}$ under this isomorphism.

0.6. Twisted actions. We follow the approach in [10, Section 3]. Let $\sigma$ be an automorphism on $G$ such that $\sigma(B)=B$ and $\sigma(T)=T$. We also assume that $\sigma$ is a diagram automorphism, i.e., the order of $\sigma$ coincides with the order of the associated permutation on $I$.

By [20, page 55], we may assume that $\dot{w}=\sigma(\dot{w})$ for $w \in W^{\sigma}$.

Let $G_{\sigma}$ (resp. $X_{\sigma}$ ) be the $(G \times G)$-variety which as a variety is isomorphic to $G$ (resp. $X)$, but the $G \times G$-action is twisted by $\left(g, g^{\prime}\right) \mapsto\left(g, \sigma\left(g^{\prime}\right)\right)$. Then $G_{\sigma}$ is an open $G \times G$-subvariety of $X_{\sigma}$ and we call $X_{\sigma}$ the wonderful compactification of $G_{\sigma}$.

Under the natural bijection between $X$ and $X_{\sigma}$, we may identify the $G \times G$-orbits on $X$ with the $G \times G$-orbits on $X_{\sigma}$. We denote by $Z_{J, \sigma}$ the $G \times G$-orbit on $X_{\sigma}$ that corresponds to $Z_{\sigma(J)} \subset X$. Accordingly, we denote by $h_{J, \sigma}$ the base point in $Z_{J, \sigma}$ which corresponds to the base point $h_{\sigma(J)}$ of $Z_{\sigma(J)}$.

0.7. $\sigma$-semisimple elements in $G_{\sigma}$. We follow the notation of [17]. An element $g \in G_{\sigma}$ is called $\sigma$-semisimple if it is conjugated to an element in $T$. We have the following result.

Theorem 0.1. Let $g \in G_{\sigma}$. Then the following conditions are equivalent:

(1) The element $g$ is $\sigma$-semisimple.

(2) The $G$-orbit of $g$ is closed in $G_{\sigma}$.

In this case, the isotropy subgroup of $g$ in $G$ is reductive.

The equivalence of (1) and (2) can be found in [11, 1.4 (e)] (in terms of disconnected groups instead of twisted conjugation action). In the case of a simply connected group, the equivalence is also proved in [17, Proposition 3]. By Fact 3, Matsushima's criterion, the $G$-orbit of $g$ is closed implies that the isotropy subgroup of $g$ is reductive.

0.8. $G$-stable-piece decomposition. Let $G_{\Delta}$ be the diagonal image of $G$ in $G \times$ $G$. The classification of the $G_{\Delta}$-orbits on $X$ was obtained by Lusztig [12] in terms of $G$-stable pieces. A similar result also occurs in [6]. We list some known results which will be used later.

For $J \subset I$ and $w \in W^{\sigma(J)}$, set

$$
Z_{J, \sigma ; w}=G_{\Delta}(B \dot{w}, B) \cdot h_{J, \sigma} .
$$

We call $Z_{J, \sigma ; w}$ a $G$-stable piece of $X_{\sigma}$. By [12, 12.3] and [7, Proposition 2.6], $X_{\sigma}$ is a disjoint union of the $G$-stable pieces.

Fact 5. $X_{\sigma}=\bigsqcup_{J \subset I} \bigsqcup_{w \in W^{\sigma(J)}} Z_{J, \sigma ; w}$. 
Set $I(J, \sigma ; w)=\max \{K \subset J \mid w \sigma(K)=K\}$. Then the subvariety $L_{I(J, \sigma ; w)} \dot{w}$ of $G_{\sigma}$ is stable under the action of $L_{I(J, \sigma ; w)} \times L_{I(J, \sigma ; w)}$ and, in particular, is stable under the conjugation action of $L_{I(J, \sigma ; w)}$. Moreover, by [12, 12.3(a)] and [8, Lemma $1.4]$,

$$
Z_{J, \sigma ; w}=G_{\Delta}\left(L_{I(J, \sigma ; w)} \dot{w}, 1\right) \cdot h_{J, \sigma}
$$

and there exists a natural bijection between the $G_{\Delta}$-orbits on $Z_{J, \sigma ; w}$ and the $L_{I(J, \sigma ; w)}$-orbits on $L_{I(J, \sigma ; w)} \dot{w} / Z\left(L_{J}\right) \subset G_{\sigma} / Z\left(L_{J}\right)$ (for the conjugation action of $\left.L_{I(J, \sigma ; w)}\right)$.

For any point $z$ in $Z_{J, \sigma ; w}$, the isotropy subgroup

$$
G_{z}=\{g \in G \mid(g, g) \cdot z=z\}
$$

was described explicitly in [6. Theorem 3.13]. In particular, if $z \in Z_{J, \sigma ; w}$ with $w \neq 1$, then $G_{z}$ is not reductive (since $\psi\left(n_{v}\right)$ in loc. cit. is contained in the nilpotent radical of the Lie algebra of $G_{z}$ and by the explicit expression, $\left.\psi\left(n_{v}\right) \neq 0\right)$. For $z=(l, 1) \cdot h_{J, \sigma}$ with $l \in L_{I(J, \sigma ; 1)}, G_{z} \subset L_{I(J, \sigma ; 1)}$ (see loc. cit., see also [9, 3.4]). Now the following fact follows from the known result on the centralizer of $\sigma$-semisimple elements in reductive groups.

Fact 6. Let $z=(g l \dot{w}, g) \cdot h_{J, \sigma}$ for $g \in G$ and $l \in L_{I(J, \sigma ; w)}$. Then $G_{z}$ is reductive if and only if $w=1$ and $l$ is a $\sigma$-semisimple element in $L_{I(J, \sigma ; 1)}$.

By [8. Theorem 4.5], the closure of each $G$-stable piece is a union of $G$-stable pieces and the closure relation can be described explicitly. More precisely, for $J \subset I, w \in W^{\sigma(J)}$ and $w^{\prime} \in W$, we write $w^{\prime} \leqslant_{J, \sigma} w$ if there exists $u \in W_{J}$ such that $w^{\prime} \geqslant u w \sigma(u)^{-1}$. Then

$$
\overline{Z_{J, \sigma ; w}}=\bigsqcup_{J^{\prime} \subset J} \bigsqcup_{w^{\prime} \in W^{\sigma\left(J^{\prime}\right)}, w^{\prime} \leqslant J, \sigma} Z_{J^{\prime}, \sigma ; w^{\prime}} .
$$

Notice that if $1 \leqslant J_{, \sigma} w$, then we must have $w=1$. Therefore,

Fact 7. $\bigsqcup_{J \subset I} Z_{J, \sigma ; 1}$ is open in $X_{\sigma}$.

0.9. Nilpotent Cone of $X$. Let $G_{\text {sc }}$ be a simply connected covering of $G$. The diagram automorphism $\sigma$ of $G$ can be lifted to an automorphism of $G_{\mathrm{sc}}$, which we still denote by $\sigma$ (see [16, Chapter 9]).

For any dominant weight $\lambda$, let $\mathrm{H}(\lambda)$ be the dual Weyl module for $G_{\mathrm{sc}}$ with lowest weight $-\lambda$. Let ${ }^{\sigma} \mathrm{H}(\lambda)$ be the $G_{\mathrm{sc}}$-module which as a vector space is $\mathrm{H}(\lambda)$, but the $G_{\mathrm{sc}}$-action is twisted by the automorphism $\sigma$ on $G_{\mathrm{sc}}$. Then there exists (up to a nonzero constant) a unique $G_{\text {sc }}$ isomorphism ${ }^{\sigma} \mathrm{H}(\lambda) \rightarrow \mathrm{H}(\sigma(\lambda))$. In particular, if $\lambda=\sigma(\lambda)$, then we have an isomorphism $f_{\lambda}:{ }^{\sigma} \mathrm{H}(\lambda) \rightarrow \mathrm{H}(\lambda)$.

By [5, 3.9], there exists a $G \times G$-equivariant morphism

$$
\rho_{\lambda}: X \rightarrow \mathbb{P}(\operatorname{End}(\mathrm{H}(\lambda)))
$$

which extends the morphism $G \rightarrow \mathbb{P}(\operatorname{End}(\mathrm{H}(\lambda)))$ defined by $g \mapsto g\left[\operatorname{Id}_{\lambda}\right]$, where $\left[\mathrm{Id}_{\lambda}\right]$ denotes the class representing the identity map on $\mathrm{H}(\lambda)$ and $g$ acts by the left action. We denote by $\mathcal{L}_{X}(\lambda)$ the $G_{\mathrm{sc}} \times G_{\mathrm{sc}}$-linearized invertible sheaf on $X$ which is the pullback under $\rho_{\lambda}$ of $\mathcal{O}(1)$ with its canonical linearization. This is the "usual" linearized invertible sheaf on $X$ associated to the weight $\lambda$, e.g., as defined in [2, p. 100].

The morphism $\rho_{\lambda}$ induces a $G \times G$-equivariant morphism

$$
X_{\sigma} \rightarrow \mathbb{P}\left(\operatorname{Hom}\left({ }^{\sigma} \mathrm{H}(\lambda), \mathrm{H}(\lambda)\right)\right) \text {. }
$$


When $\lambda=\sigma(\lambda)$, we may apply the isomorphism $f_{\lambda}:{ }^{\sigma} \mathrm{H}(\lambda) \rightarrow \mathrm{H}(\lambda)$ to obtain the $G \times G$-equivariant morphism

$$
\rho_{\lambda, \sigma}: X_{\sigma} \rightarrow \mathbb{P}(\operatorname{End}(\mathrm{H}(\lambda))) .
$$

As above, $\mathcal{L}_{X_{\sigma}}(\lambda)$ denotes the $G_{\mathrm{sc}} \times G_{\mathrm{sc}}$-linearized invertible sheaf on $X_{\sigma}$ which is the pullback under $\rho_{\lambda, \sigma}$ of $\mathcal{O}(1)$ with its canonical linearization. Of course $X_{\sigma}$ equals $X$ as varieties, and $\mathcal{L}_{X_{\sigma}}(\lambda)$ equals $\mathcal{L}_{X}(\lambda)$ as invertible sheaves on this variety. But the $G_{\mathrm{sc}} \times G_{\mathrm{sc}}$-actions are not the same, and thus the $G_{\mathrm{sc}} \times G_{\mathrm{sc}}$-linearized invertible sheaves are not the same.

For sufficiently divisible and positive $n$, the $G_{\mathrm{sc}} \times G_{\mathrm{sc}}$-linearization of $\mathcal{L}_{X_{\sigma}}(\lambda)^{\otimes n}=$ $\mathcal{L}_{X_{\sigma}}(n \cdot \lambda)$ factors through a $G \times G$-linearization. This induces a $G_{\Delta}$-linearization of $\mathcal{L}_{X_{\sigma}}(\lambda)^{\otimes n}$. If, moreover, $\lambda$ is regular, then $\mathcal{L}_{X_{\sigma}}(\lambda)$ is ample (see [21, section 2]).

For $\lambda=\sigma(\lambda) \neq 0$, let $\mathcal{N}(\lambda)_{\sigma}$ be the subvariety of $X_{\sigma}$ consisting of elements in $\mathbb{P}(\operatorname{End}(\mathrm{H}(\lambda)))$ that are represented by a nilpotent endomorphism of $\mathrm{H}(\lambda)$. We call $\mathcal{N}(\lambda)_{\sigma}$ the nilpotent cone of $X_{\lambda}$ associated to the dominant weight $\lambda$. We have an explicit description of $\mathcal{N}(\lambda)$ which was obtained in [10, Proposition 4.4]

$$
\mathcal{N}(\lambda)_{\sigma}=\bigsqcup_{J \subset I} \bigsqcup_{\substack{w \in W^{\sigma(J)} \\ I(\lambda) \cap \operatorname{supp}(w) \neq \varnothing}} Z_{J, \sigma ; w},
$$

where $I(\lambda)=\left\{i \in I \mid a_{i} \neq 0\right\}$ for $\lambda=\sum_{i \in I} a_{i} \omega_{i}$ and $\operatorname{supp}(w) \subset I$ is the set of simple roots whose associated simple reflections occur in some (or equivalently, any) reduced decomposition of $w$.

Two subvarieties of $X$ related to the nilpotent cones of $X$ are of special interest. One is

$$
\bigcap_{\lambda \text { is dominant }, \lambda=\sigma(\lambda) \neq 0} \mathcal{N}(\lambda)_{\sigma}=\bigsqcup_{J \subset I} \bigsqcup_{w \in W^{\sigma(J)}, \operatorname{supp}_{\sigma}(w)=I} Z_{J, \sigma ; w} ;
$$

here $\operatorname{supp}_{\sigma}(w)$ is the minimal $\sigma$-stable subset of $I$ containing $\operatorname{supp}(w)$. This subvariety is actually the boundary of the closure in $X_{\sigma}$ of unipotent subvariety of $G_{\sigma}$ in the case where $G$ is simple (See [7, Theorem 4.3] and [10, Theorem 7.3]).

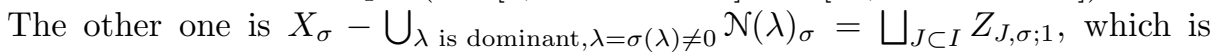
the complement of $\mathcal{N}(\lambda)_{\sigma}$ for any $\sigma$-stable dominant regular weight. By the next theorem, this subvariety is actually the semistable locus of $X_{\sigma}$ for the $G_{\Delta}$-action.

Theorem 0.2. Suppose that $\lambda$ is a $\sigma$-stable, dominant and regular weight and the $G_{\mathrm{sc}} \times G_{\mathrm{sc}}$-linearization of $\mathcal{L}_{X_{\sigma}}(\lambda)=\mathcal{L}_{X_{\sigma}}(n \cdot \lambda)$ induces a $G_{\Delta}$-linearization on $\mathcal{L}_{X_{\sigma}}(\lambda)$. Then the semistable locus $\left(X_{\sigma}\right)^{s s}\left(\mathcal{L}_{X_{\sigma}}(\lambda)\right)$ equals $\bigsqcup_{J \subset I} Z_{J, \sigma ; 1}$. In particular, the semistable locus is independent of the choice of weight $\lambda$.

Proof. We simply write the semistable locus $\left(X_{\sigma}\right)^{\text {ss }}\left(\mathcal{L}_{X_{\sigma}}(\lambda)\right)$ as $X_{\sigma}^{\text {ss }}$. On $\operatorname{End}(H(\lambda))$ the characteristic polynomial map

$$
\chi: \operatorname{End}(H(\lambda)) \rightarrow k[t], \quad(f: H(\lambda) \rightarrow H(\lambda)) \mapsto \chi_{f}(t)
$$

is a morphism which is invariant under the conjugation action. The coefficients of the characteristic polynomial define homogeneous polynomials on $\operatorname{End}(H(\lambda))$ which are invariant under the conjugation action. Also, the degree is positive except for the leading coefficient (which is 1 ). Thus each nonleading coefficient defines a $G_{\Delta}$-invariant sections of positive power $\mathcal{O}(n)$ on $\mathbb{P}(\operatorname{End}(H(\lambda)))$. The pullbacks of these sections are $G_{\Delta}$-invariant sections of positive powers $\mathcal{L}_{X_{\sigma}}(\lambda)^{\otimes n}$. By Fact 1 , 
the nonvanishing locus of each of these sections is in the semistable locus. Equivalently, the nonsemistable locus is contained in the common zero locus of all of these sections. But the common zero locus of these pullback sections on $X_{\sigma}$ equals the inverse image of the common zero locus of the original sections on $\mathbb{P}(\operatorname{End}(H(\lambda)))$; and this common zero locus is precisely the nilpotent cone in $\mathbb{P}(\operatorname{End}(H(\lambda)))$. Thus the nonsemistable locus is contained in $\mathcal{N}(\lambda)_{\sigma}$. So $X_{\sigma}^{\text {ss }}$ contains $X_{\sigma}-\mathcal{N}(\lambda)_{\sigma}$, i.e., $X_{\sigma}^{\text {ss }}$ contains $\bigsqcup_{J \subset I} Z_{J, \sigma ; 1}$.

Also, by Fact $7, X_{\sigma}^{s s}-\bigsqcup_{J \subset I} Z_{J, \sigma ; 1}$ is closed in $X_{\sigma}^{s s}$. If $X_{\sigma}^{s s}$ strictly contains $\bigsqcup_{J \subset I} Z_{J, \sigma ; 1}$, then there exists a closed $G_{\Delta}$-orbit in $X_{\sigma}^{s s}$ that is not contained in $\bigsqcup_{J \subset I} Z_{J, \sigma ; 1}$. Let $z$ be an element in that orbit. By Fact 3 above, the isotropy subgroup of $z,\{g \in G \mid(g, g) \cdot z=z\}$, is reductive. By Fact 5 above, $z$ is in $Z_{J, \sigma ; w}$ for some $J \subset I$ and $w \in W^{\sigma(J)}$ with $w \neq 1$; but this contradicts Fact 6 above. Therefore, $X_{\sigma}^{s s}$ equals $\bigsqcup_{J \subset I} Z_{J, \sigma ; 1}$.

In the rest of this paper, we study the geometric quotient $X_{\sigma} / / G$. It uses some detailed analysis on the structure of the semistable locus in 9 . It is worth mentioning that $\left[9\right.$ only uses the description of $X_{\sigma}^{\text {ss }}$ of this paper and no logical loop appears.

The automorphism $\sigma$ on $G$ can be extended in a natural way to an automorphism on $X$, which we still denote by $\sigma$. This automorphism sends $\left(g, g^{\prime}\right) \cdot h_{J}$ to $\left(\sigma(g), \sigma\left(g^{\prime}\right)\right) \cdot h_{\sigma(J)}$. Now we have the following result.

Lemma 0.3. Let $\bar{T}$ be the closure of $T$ in $X$ and $\bar{T}^{\sigma}$ be the subset of $\sigma$-fixed points. Then

$$
\bar{T}^{\sigma}=\bigsqcup_{J \subset I, \sigma(J)=J}\left(N(T)^{\sigma}\right)_{\Delta} \cdot\left(T^{\sigma}, 1\right) h_{J} .
$$

In particular, $\bar{T}^{\sigma}$ is the closure of $T^{\sigma}$ in $X$.

Proof. By [1, Lemma 6.1.6 (ii)],

$$
\bar{T}=\bigsqcup_{J \subset I} N(T)_{\Delta} \cdot(T, 1) \cdot h_{J}=\sqcup_{J \subset I, w \in W^{J}}(\dot{w} t, \dot{w}) \cdot h_{J} .
$$

It is easy to see that $\bigsqcup_{J \subset I, \sigma(J)=J}\left(N(T)^{\sigma}\right)_{\Delta} \cdot\left(T^{\sigma}, 1\right) h_{J} \subset \bar{T}^{\sigma}$. Let $z=(\dot{w} t, \dot{w}) \cdot h_{J}$ for some $w \in W^{J}, t \in T$ and $J \subset I$. Then $\sigma(z)=(\sigma(\dot{w}) \sigma(t), \sigma(\dot{w})) \cdot h_{\sigma(J)}$. If $z=\sigma(z)$, then $J=\sigma(J)$ and $(\dot{w} t, \dot{w}) \cdot h_{J}=(\sigma(\dot{w}) \sigma(t), \sigma(\dot{w})) \cdot h_{J}$. In particular, $(B \dot{w}, B \dot{w}) \cdot h_{J}=(B \sigma(\dot{w}), B \sigma(\dot{w})) \cdot h_{J}$, here $w, \sigma(w) \in W^{J}$. By [18, Proposition $1(\mathrm{i})], w=\sigma(w)$.

So $(t, 1) \cdot h_{J}=(\sigma(t), 1) \cdot h_{J}$. It is easy to see that $(T, 1) \cdot h_{J} \cong T / Z\left(L_{J}\right)$ and the natural projection $T^{\sigma} \rightarrow\left(T / Z\left(L_{J}\right)\right)^{\sigma}$ is surjective. Hence $(t, 1) \cdot h_{J}=\left(t^{\prime}, 1\right) \cdot h_{J}$ for some $t^{\prime} \in T^{\sigma}$. Thus $\bar{T}^{\sigma} \subset \bigsqcup_{J \subset I, \sigma(J)=J}\left(N(T)^{\sigma}\right)_{\Delta} \cdot\left(T^{\sigma}, 1\right) h_{J}$.

Let $\overline{T^{\sigma}}$ be the closure of $T^{\sigma}$ in $X$. For any $J \subset I$, by [18, Lemma 1(i)], $h_{J}$ is contained in the closure of $\left\{\left(\Pi_{j \notin J} \omega_{j}^{\vee}(a) ; a \in k^{\times}\right\}\right.$. Thus $h_{J} \subset \overline{T^{\sigma}}$. Thus $\left(T^{\sigma}, 1\right)$. $h_{J} \subset \overline{T^{\sigma}}$ if $\sigma(J)=J$. Since $\left(N(T)^{\sigma}\right)_{\Delta} \cdot T^{\sigma}=T^{\sigma}$, we have that $\left(N(T)^{\sigma}\right)_{\Delta} \cdot \overline{T^{\sigma}}=\overline{T^{\sigma}}$ and $\left(N(T)^{\sigma}\right)_{\Delta} \cdot\left(T^{\sigma}, 1\right) h_{J} \subset \overline{T^{\sigma}}$. Hence $\bar{T}^{\sigma} \subset \overline{T^{\sigma}}$. On the other hand, $\bar{T}^{\sigma}$ is a closed variety of $X$ that contains $T^{\sigma}$. Hence $\bar{T}^{\sigma}=\overline{T^{\sigma}}$.

Lemma 0.4. Let $i: X \rightarrow X_{\sigma}$ be the natural bijection in $\S 0.6 . A G_{\Delta}$-orbit $\mathcal{O}$ in $X_{\sigma}^{s s}$ is closed in $X_{\sigma}^{s s}$ if and only if it intersects $i\left(\bar{T}^{\sigma}\right)$. 
Proof. Let $z \in X_{\sigma}^{\mathrm{ss}}$ such that $G_{\Delta} \cdot z$ is closed in $X_{\sigma}^{\text {ss }}$. Then $G_{\Delta} \cdot z$ is affine and $G_{z}$ is reductive. By Fact $6, z$ is of the form $(g l, g) \cdot h_{J, \sigma}$ for some $g \in G$ and some $\sigma$ semisimple element $l \in L_{I(J, \sigma ; 1)}$. So $l$ is $\sigma$-conjugate to an element in $T$. Therefore $G_{\Delta} \cdot z \cap i(\bar{T}) \neq \emptyset$. Without loss of generality, we may assume that $z=(t, 1) \cdot h_{J, \sigma}$ for some $t \in T$.

If $J \neq \sigma(J)$, then there exists $j \in \sigma(J)$, but $j \notin J$. Then

$$
\left(\omega_{j}^{\vee}(a) t, \omega_{j}^{\vee}(a)\right) \cdot h_{J, \sigma}=\left(\omega_{j}^{\vee}(a) t, 1\right) \cdot h_{J, \sigma} \in G_{\Delta} \cdot z
$$

for all $a \in k^{\times}$. By [18, Lemma 1(i)], $h_{\sigma(J)-\{j\}}$ is contained in the closure of $\left\{\left(\omega_{j}^{\vee}(a), 1\right) \cdot h_{\sigma(J)} ; a \in k^{\times}\right\}$. Thus $(t, 1) \cdot h_{J-\left\{\sigma^{-1}(j)\right\}, \sigma}$ is contained in the closure of $\left\{\left(\omega_{j}^{\vee}(a) t, 1\right) \cdot h_{J, \sigma} ; a \in k^{\times}\right\}$. That contradicts our assumption that $G_{\Delta} \cdot z$ is closed in $X_{\sigma}^{\mathrm{ss}}$.

So we must have that $J=\sigma(J)$. Notice that $t=t^{\prime} t^{\prime \prime} \sigma\left(t^{\prime}\right)^{-1}$ for some $t^{\prime} \in T$ and $t^{\prime \prime} \in T^{\sigma}$. Thus $G_{\Delta} \cdot z \cap\left(T^{\sigma}, 1\right) \cdot h_{J, \sigma} \neq \emptyset$. By the previous lemma, $i\left(\bar{T}^{\sigma}\right)=$ $\bigsqcup_{J \subset I, \sigma(J)=J}\left(N(T)^{\sigma}\right)_{\Delta} \cdot\left(T^{\sigma}, 1\right) h_{J, \sigma}$. So $G_{\Delta} \cdot z \cap i\left(\bar{T}^{\sigma}\right) \neq \emptyset$.

On the other hand, if $z=(t, 1) \cdot h_{J, \sigma}$ for some $J=\sigma(J)$ and $t \in T^{\sigma}$, we will prove that $G_{\Delta} \cdot z$ is closed in $X_{\sigma}^{\text {ss. }}$. By $[9,3.4]$, the map $(g, l \sigma) \mapsto(g l, g) \cdot h_{J, \sigma}$ gives an isomorphism from $G \times_{L_{J}} L_{J} \sigma / Z\left(L_{J}\right)$ to $Z_{J, \sigma ; 1}$. By Theorem 0.1 , $\left(L_{J}\right)_{\Delta} \cdot z$ is closed in $\left(L_{J}, 1\right) \cdot h_{J, \sigma} \cong L_{J} \sigma / Z\left(L_{J}\right)$. Therefore $G_{\Delta} \cdot z$ is closed in $Z_{J, \sigma ; 1}$. Now the statement follows from the next lemma.

Lemma 0.5. Let $z=(t, 1) \cdot h_{J, \sigma}$ for some $t \in T^{\sigma}$ and $J \subset I$ with $\sigma(J)=J$. Then $\overline{G_{\Delta} \cdot z} \cap X_{\sigma}^{s s} \subset Z_{J, \sigma ; 1}$.

Proof. Let $\bar{B}$ be the closure of $B$ in $X_{\sigma}$. Consider the proper map $f: G \times{ }_{B} \bar{B} \rightarrow X_{\sigma}$, $(g, b) \mapsto(g, g) \cdot b$. Its restriction $G \times_{B}\left(\bar{B} \cap X_{\sigma}^{\text {ss }}\right) \rightarrow X_{\sigma}^{\text {ss }}$ is still proper. Notice that $B_{\Delta} \cdot z \subset \bar{B}$. Thus $\overline{G_{\Delta} \cdot z}=G_{\Delta} \cdot \overline{B_{\Delta} \cdot z}$ and $\overline{G_{\Delta} \cdot z} \cap X_{\sigma}^{\text {ss }}=G_{\Delta} \cdot\left(\overline{B_{\Delta} \cdot z} \cap X_{\sigma}^{\text {ss }}\right)$. It remains to prove that $\overline{B_{\Delta} \cdot z} \cap X_{\sigma}^{\text {ss }} \subset Z_{J, \sigma ; 1}$.

Let $U$ be the unipotent radical of $B$ and let $U^{-}$be the unipotent radical of $B^{-}$. Set $Y=\bigsqcup_{K \subset I}(T, 1) \cdot h_{K, \sigma}$. For any $w \in W^{\sigma}$, set $Y_{w}=\bigsqcup_{K \subset I}(B \dot{w}, B \dot{w}) \cdot h_{K, \sigma}$.

By [9, Theorem $2.3 \&$ Lemma 2.5], $\bar{B} \cap X_{\sigma}^{\text {ss }}=\bigcup_{w \in W^{\sigma}} Y_{w}$ and for any $w \in W^{\sigma}$, $Y_{w}$ is open in $\bar{B} \cap X_{\sigma}^{\mathrm{ss}}$ and stable under the diagonal action of $B$. To show that $\overline{B_{\Delta} \cdot z} \cap X_{\sigma}^{\text {ss }} \subset Z_{J, \sigma ; 1}$, it suffices to prove that $B_{\Delta} \cdot z$ is closed in $Y_{w}$ for all $w \in W^{\sigma}$ such that $z \in Y_{w}$.

Let $w \in W^{\sigma}$ with $z=(t, 1) \cdot h_{J, \sigma} \in Y_{w}$. Then $z \in(T \dot{w}, T \dot{w}) \cdot h_{J, \sigma}$. Thus $w \in W_{J}$. Set $z^{\prime}=\left(\dot{w}^{-1}, \dot{w}^{-1}\right) \cdot z=\left(\dot{w}^{-1} t \dot{w}, 1\right) \cdot h_{J, \sigma}$.

By [9, Proof of Lemma 2.5], for any $t^{\prime} \in T$ and $K \subset I$,

$$
\left(U t^{\prime} \dot{w}, U \dot{w}\right) \cdot h_{K, \sigma}=\left(\left(U \cap \dot{w} U \dot{w}^{-1}\right) t^{\prime} \dot{w},\left(U \cap \dot{w} U^{-} \dot{w}^{-1}\right) \dot{w}\right) \cdot h_{K, \sigma}
$$

and the map

$$
\left(U \cap \dot{w} U \dot{w}^{-1}\right) \times\left(U \cap \dot{w} U^{-} \dot{w}^{-1}\right) \times Y \rightarrow Y_{w}
$$

defined by $\left(u, u^{\prime}, z_{1}\right) \mapsto\left(u \dot{w}, u^{\prime} \dot{w}\right) \cdot z_{1}$, is an isomorphism.

By (a),

$$
\left.B_{\Delta} \cdot z \subset(U, U) T_{\Delta} \cdot z=\left(\left(U \cap \dot{w} U \dot{w}^{-1}\right) \dot{w},\left(U \cap \dot{w} U^{-} \dot{w}^{-1}\right) \dot{w}\right)\right) T_{\Delta} \cdot z^{\prime} .
$$

By (b), $\left.\overline{B_{\Delta} \cdot z} \cap Y_{w} \subset\left(\left(U \cap \dot{w} U \dot{w}^{-1}\right) \dot{w},\left(U \cap \dot{w} U^{-} \dot{w}^{-1}\right) \dot{w}\right)\right)\left(\overline{T_{\Delta} \cdot z^{\prime} \cap Y}\right)$. Hence $T_{\Delta} \cdot z^{\prime} \cap Y \neq \emptyset$. 
As in the proof of [9. Lemma 3.1], the map $T \rightarrow k$ defined by $a \mapsto \alpha_{i}(a)$ extends in a unique way to $\tilde{\alpha}_{i}: Y \rightarrow k$ and

$$
\tilde{\alpha}_{i}\left((a, 1) \cdot h_{K, \sigma}\right)= \begin{cases}\alpha_{i}(a), & \text { if } i \in \sigma(K), \\ 0, & \text { otherwise } .\end{cases}
$$

Set $Y^{\prime}=\left\{z^{\prime} \in Y ; \prod_{i \in \mathcal{O}} \tilde{\alpha}_{i}\left(z^{\prime}\right)=1\right.$ for all $\sigma-$ orbit $\mathcal{O}$ of $J, \tilde{\alpha}_{i}\left(z^{\prime}\right)=0$ for all $\left.i \notin J\right\}$. Then $Y^{\prime}$ is closed in $Y$ and $Y^{\prime} \subset(T, 1) \cdot h_{J, \sigma}$.

Set $T^{\prime}=\left\{a \sigma(a)^{-1} ; a \in T\right\}$. Then $T_{\Delta} \cdot z^{\prime}=\left(\dot{w}^{-1} t \dot{w} T^{\prime}, 1\right) \cdot h_{J, \sigma}$ and $\left(T^{\prime}, 1\right) \cdot h_{J, \sigma} \subset$ $Y^{\prime} \subset(T, 1) \cdot h_{J, \sigma}$. Since $\left(T^{\prime}, 1\right) \cdot h_{J, \sigma}$ is closed in $(T, 1) \cdot h_{J, \sigma}$, it is also closed in $Y^{\prime}$. Thus $\left(T^{\prime}, 1\right) \cdot h_{J, \sigma}$ is closed in $Y$ and $T_{\Delta} \cdot z^{\prime}$ is also closed in $Y$. Hence $\overline{T_{\Delta} \cdot z^{\prime}} \cap Y=T_{\Delta} \cdot z^{\prime}$ and $\overline{G_{\Delta} \cdot z} \cap X_{\sigma}^{\mathrm{ss}} \subset Z_{J, \sigma ; 1}$.

Lemma 0.6. Set $\tilde{N}^{\sigma}=\left\{n \in G ; n T^{\sigma} \sigma(n)^{-1}=T^{\sigma}\right\} \supset N(T)^{\sigma}$ and $\tilde{W}=\tilde{N}^{\sigma} / T^{\sigma}$. Then $\tilde{W}$ is a finite group and the diagonal action of $\tilde{N}^{\sigma}$ on $i\left(\bar{T}^{\sigma}\right)$ induces an action of $\tilde{W}$ on $i\left(\bar{T}^{\sigma}\right)$. Moreover, for every element $z \in i\left(\bar{T}^{\sigma}\right)$, the intersection $G_{\Delta} \cdot z \cap i\left(\bar{T}^{\sigma}\right) \subset X_{\sigma}$ of the $G_{\Delta}$-orbit with $i\left(\bar{T}^{\sigma}\right)$ equals the $\tilde{W}$-orbit $\tilde{W}_{\Delta} \cdot z$.

Proof. Since $\sigma$ permutes the basis $\left\{\omega_{i}^{\vee}\right\}$ of the coweight lattice, $T^{\sigma}$ is connected. By [11, 1.14(a)], $\tilde{W}$ is a finite group. By Lemma $0.3, i\left(\bar{T}^{\sigma}\right)$ is the closure of $i\left(T^{\sigma}\right)$ in $X_{\sigma}$. Thus the diagonal action of $\tilde{N}^{\sigma}$ on $i\left(T^{\sigma}\right)$ extends in a unique way to an action on $i\left(\bar{T}^{\sigma}\right)$. Since $T^{\sigma}$ acts trivially on $i\left(\bar{T}^{\sigma}\right)$, the diagonal action of $\tilde{N}^{\sigma}$ on $i\left(\bar{T}^{\sigma}\right)$ induces an action of $\tilde{W}$ on $i\left(\bar{T}^{\sigma}\right)$.

It remains to prove that if $z, z^{\prime} \in i\left(\bar{T}^{\sigma}\right)$ are in the same $G_{\Delta}$-orbit, then $z^{\prime} \in$ $\tilde{W}_{\Delta} \cdot z$. By Lemma $0.3, i\left(\bar{T}^{\sigma}\right)=\tilde{W}_{\Delta} \cdot \bigsqcup_{J \subset I, \sigma(J)=J}\left(T^{\sigma}, 1\right) h_{J, \sigma}$. We may assume that $z=(t, 1) \cdot h_{J, \sigma}$ and $z^{\prime}=\left(t^{\prime}, 1\right) \cdot h_{J, \sigma}$, where $t, t^{\prime} \in T^{\sigma}$ and $J=\sigma(J)$. Again by [9, 3.4], $Z_{J, \sigma ; 1} \cong G \times_{L_{J}} L_{J} \sigma / Z\left(L_{J}\right)$. So $t$ and $t^{\prime}$ are in the same $\sigma$-conjugacy class of $G_{J}$. In other words, there exists $g \in G$ such that $g t \sigma(g)^{-1}=t^{\prime} z$ for some $z \in Z\left(L_{J}\right)$.

Notice that $Z\left(L_{J}\right) \subset T$ is a connected torus. By [11, 1.2(a)], there exists $z_{1} \in$ $Z\left(L_{J}\right)$ and $z_{2} \in Z\left(L_{J}\right)^{\sigma} \subset T^{\sigma}$ such that $z=z_{1}^{-1} z_{2} \sigma\left(z_{1}\right)$. Then $z_{1} g t \sigma\left(z_{1} g\right)^{-1}=t^{\prime} z_{2}$. By [11, 1.14(d)], there exists $n \in L_{J}$ with $n T^{\sigma} \sigma(n)^{-1}=T^{\sigma}$ and $n t \sigma(n)^{-1}=t^{\prime} z_{2}$. Hence $z^{\prime}=\left(t^{\prime}, 1\right) \cdot h_{J, \sigma}=\left(t^{\prime} z_{2}, 1\right) \cdot h_{J, \sigma} \in \tilde{W}_{\Delta} \cdot z$.

Theorem 0.7. The embedding $i\left(\bar{T}^{\sigma}\right) \hookrightarrow X_{\sigma}$ induces an morphism

$$
\bar{i}: i\left(\bar{T}^{\sigma}\right) / \tilde{W} \rightarrow X_{\sigma} / / G
$$

which is a bijection on points. If char $(k)$ equals $0, \bar{i}$ is an isomorphism. Also, if $\sigma$ is the identity map (and char $(k)$ is arbitrary), then $\bar{T}^{\sigma} / \tilde{W}$ equals $\bar{T} / W$ and the induced morphism

$$
\bar{i}: \bar{T} / W \rightarrow X / / G
$$

is an isomorphism.

Proof. The morphism $i\left(\bar{T}^{\sigma}\right) \rightarrow X_{\sigma}^{s s} \rightarrow X_{\sigma} / / G$ is $\tilde{W}$-invariant, and hence factors through a morphism $\bar{i}: i\left(\bar{T}^{\sigma}\right) / \tilde{W} \rightarrow X_{\sigma} / / G$. By Lemma 0.4 . every closed $G$-orbit in $X_{\sigma}^{\text {ss }}$ intersects $i\left(\bar{T}^{\sigma}\right)$. Thus $i$ is surjective. For every element $z$ in $i\left(\bar{T}^{\sigma}\right)$, the $G$-orbit of $z$ is closed in $X_{\sigma}^{\text {ss }}$. Thus two elements $z, z^{\prime}$ in $i\left(\bar{T}^{\sigma}\right)$ have the same image under $\bar{i}$ if and only if they lie in the same $G$-orbit. On the other hand, by Lemma 0.6. two elements $z, z^{\prime}$ in $i\left(\bar{T}^{\sigma}\right)$ lie in the same $G$-orbit if and only if they lie in the same $\tilde{W}$-orbit. Hence $\bar{i}$ is a bijection on points. 
Assume for the moment that $\operatorname{char}(k)$ is 0 . By Fact $4, X_{\sigma}^{\mathrm{ss}}$ is normal. And $\bar{i}$ is a dominant morphism of varieties which is a bijection on points. Since $\operatorname{char}(k)=0$, this implies that $\bar{i}$ is birational. By Zariski's Main Theorem, a bijective birational morphism of varieties is an isomorphism if the target is normal. Thus $\bar{i}$ is an isomorphism when $\operatorname{char}(k)$ is 0 .

Next, assume that $\sigma$ is the identity map, but $\operatorname{char}(k)$ may be arbitrary. Then $\bar{T}^{\sigma}=\bar{T}$ and $\tilde{W}=W$. In this case, $\bar{T}^{\sigma} / \tilde{W}$ equals $\bar{T} / W$ for the natural $W$-action on $\bar{T}$ which extends the $W$-action on $T$. By [19, section 6$]$, the restriction of $\bar{i}$ to the open subvariety $T / W$ of $\bar{T} / W$ gives an isomorphism $T / W \cong G / / G$. Hence, as above, $\bar{i}$ is a bijective, birational morphism of varieties whose target is a normal variety. So again by Zariski's Main Theorem, $\bar{i}$ is an isomorphism.

\section{ACKNOWLEDGMENT}

We thank the referee for a careful reading of this article and useful comments.

\section{REFERENCES}

1. Michel Brion and Shrawan Kumar, Frobenius splitting methods in geometry and representation theory, Progress in Mathematics, vol. 231, Birkhäuser Boston Inc., Boston, MA, 2005. MR.2107324 (2005k:14104)

2. Michel Brion and Patrick Polo, Large Schubert varieties, Represent. Theory 4 (2000), 97-126 (electronic). MR1789463 (2001j:14066)

3. C. De Concini, S. Kannan, and A. Maffei, The quotient of a complete symmetric variety, Mosc. Math. J. 8 (2008), 667-696. MR2499351 (2011b:14100)

4. C. De Concini and C. Procesi, Complete symmetric varieties, Invariant theory (Montecatini, 1982), Lecture Notes in Math., vol. 996, Springer, Berlin, 1983, pp. 1-44. MR718125 (85e:14070)

5. C. De Concini and T. A. Springer, Compactification of symmetric varieties, Transform. Groups 4 (1999), no. 2-3, 273-300, Dedicated to the memory of Claude Chevalley. MR 1712864 (2000f:14079)

6. Sam Evens and Jiang-Hua Lu, On the variety of Lagrangian subalgebras. II, Ann. Sci. École Norm. Sup. (4) 39 (2006), no. 2, 347-379. MR2245536 (2007d:17031)

7. Xuhua He, Unipotent variety in the group compactification, Adv. Math. 203 (2006), no. 1, 109-131. MR2231043 (2007h:20045)

8. (2007), no. 7, 3005-3024 (electronic). MR2299444 (2008c:14063)

9. Character sheaves on the semi-stable locus of a group compactification, Adv. Math. 225 (2010), no. 6, 3258-3290. MR2729008

10. Xuhua He and Jesper Funch Thomsen, Closures of Steinberg fibers in twisted wonderful compactifications, Transform. Groups 11 (2006), no. 3, 427-438. MR2264461 (2007i:14049)

11. G. Lusztig, Character sheaves on disconnected groups. I, Represent. Theory 7 (2003), 374-403 (electronic). MR2017063(2006d:20090a)

12. Marabolic character sheaves. II, Mosc. Math. J. 4 (2004), no. 4, 869-896, 981. MR 2124170 (2006d:20091b)

13. D. Mumford, J. Fogarty, and F. Kirwan, Geometric invariant theory, third ed., Ergebnisse der Mathematik und ihrer Grenzgebiete (2) [Results in Mathematics and Related Areas (2)], vol. 34, Springer-Verlag, Berlin, 1994. MR1304906 (95m:14012)

14. R. W. Richardson, Affine coset spaces of reductive algebraic groups, Bull. London Math. Soc. 9 (1977), no. 1, 38-41. MR0437549 (55:10473)

15. C. S. Seshadri, Geometric reductivity over arbitrary base, Advances in Math. 26 (1977), no. 3, 225-274. MR0466154 (57:6035)

16. T. A. Springer, Linear algebraic groups, second ed., Progress in Mathematics, vol. 9, Birkhäuser Boston, Inc., Boston, MA, 1998. MR1642713 (99h:20075)

17. 539-545. MR2264465(2007f:20081) 
18. Tonny A. Springer, Some results on compactifications of semisimple groups, International Congress of Mathematicians. Vol. II, Eur. Math. Soc., Zürich, 2006, pp. 1337-1348. MR 2275648 (2008a:14063)

19. Robert Steinberg, Regular elements of semisimple algebraic groups, Inst. Hautes Études Sci. Publ. Math. (1965), no. 25, 49-80. MR0180554 (31:4788)

20. __ Endomorphisms of linear algebraic groups, Memoirs of the American Mathematical Society, No. 80, American Mathematical Society, Providence, R.I., 1968. MR0230728 $(37: 6288)$

21. Elisabetta Strickland, A vanishing theorem for group compactifications, Math. Ann. 277 (1987), no. 1, 165-171. MR884653 (88b:14035)

Department of Mathematics, The Hong Kong University of Science and Technology, Clear Water Bay, Kowloon, Hong Kong

E-mail address: maxhhe@ust.hk

Department of Mathematics, Stony Brook University, Stony Brook, New York 11794

E-mail address: jstarr@math.sunysb.edu 